\title{
Porphyromonas gingivalis lipopolysaccharide increases lipid accumulation by affecting CD36 and ATP-binding cassette transporter A1 in macrophages
}

\author{
XIU-YING LI ${ }^{1}$, CHUN WANG ${ }^{2}$, XUE-RONG XIANG ${ }^{2}$, \\ FANG-CHUN CHEN ${ }^{2}$, CONG-MING YANG ${ }^{2}$ and $\mathrm{JING} \mathrm{WU}^{2}$ \\ ${ }^{1}$ Department of Pharmacy, The First Affiliated Hospital of Chongqing Medical University, \\ Chongqing 400010; ${ }^{2}$ Department of Periodontology, The Affiliated Hospital of Stomatology, \\ Chongqing Medical University, Chongqing 401147, P.R. China
}

Received May 10, 2013; Accepted June 19, 2013

DOI: 10.3892/or.2013.2600

\begin{abstract}
Porphyromonas gingivalis lipopolysaccharide (P. gingivalis LPS) promotes macrophage-derived foam cell formation, however, the mechanisms are not well established. In macrophages, lipid uptake is mediated by scavenger receptors including SR-A and CD36, while the cholesterol efflux is mediated by ATP-binding cassette transporter G1 (ABCG1), ABCA1 and SR-BI. We further investigated the mechanisms underlying the dysregulation by $P$. gingivalis LPS of these regulators resulting in the promotion of lipid accumulation in THP-1-derived macrophages. Our results showed that $P$. gingivalis LPS exacerbated lipid accumulation in oxidized low-density lipoprotein (oxLDL)-treated macrophages. However, cholesterol efflux was inhibited by $P$. gingivalis LPS in THP-1-derived macrophages. In oxLDL-untreated macrophages, $P$. gingivalis LPS treatment caused an increase in CD36 mRNA and protein levels, and a decrease in ABCA1 mRNA and protein levels, while having no effect on SR-A, SR-BI or ABCG1 expression. Upregulation of CD36 by $P$. gingivalis LPS resulted from activation of c-Jun/AP-1, and this was confirmed by the inhibition of increased CD36 expression after AP-1 inhibition using SP600125. However, the decreased protein stability of ABCA1 by $P$. gingivalis LPS was a result of increased calpain activity. Moreover, small hairpin RNA (shRNA) targeting heme oxygenase-1 (HO-1) augmented the $P$. gingivalis LPS-induced atherogenic effects on the expression of c-Jun/AP-1, CD36, ABCA1 and calpain activity. Accordingly, $P$. gingivalis LPS-regulated promotion of lipid accumulation in foam cells was also exacerbated by
\end{abstract}

Correspondence to: Dr Chun Wang, Department of Periodontology, The Affiliated Hospital of Stomatology, Chongqing Medical University, Chongqing 401147, P.R. China

E-mail: cqykwc@sina.com

Key words: Porphyromonas gingivalis lipopolysaccharide, cluster of differentiation 36, ATP-binding cassette transporter A1, heme oxygenase-1, activator protein-1
HO-1 shRNA. These results indicate that $P$. gingivalis LPS confers a exacerbation effect on the formation of foam cells by a novel HO-1-dependent mediation of cholesterol efflux and lipid accumulation in macrophages.

\section{Introduction}

Atherosclerotic lesions are characterized by the formation and accumulation of foam cells in the arterial intimal layer (1). Generally, foam cell formation is primarily a result of uncontrolled uptake of modified low-density lipoprotein (LDL) into the subendothelial space of macrophages $(2,3)$. In macrophages, scavenger receptors such as class A scavenger receptor (SR-A) and cluster of differentiation 36 (CD36) are reported to mediate the internalization of oxidized LDL (oxLDL) that promotes the cellular accumulation of cholesterol (4). Conversely, reverse cholesterol transporters (RCTs) including SR-BI, ATP-binding cassette transporter A1 and G1 (ABCA1 and ABCG1) are responsible for cholesterol efflux (5). Although it is known that the expression of SRs and RCTs can be affected by various atherogenic factors, such as insulin and endothelin-1 $(4,6)$, their dysregulation in the setting of atherosclerosis has not yet been fully investigated.

Porphyromonas gingivalis lipopolysaccharide ( $P$. gingivalis LPS), a major etiological agent in localized chronic periodontitis, was shown to attack the tissue enclosing the teeth, ultimately leading to tooth loss (7). Beside its role in periodontitis, increasing evidence suggests that $P$. gingivalis LPS exerts several atherogenic effects for cardiovascular diseases. For instance, $P$. gingivalis LPS triggers the secretion of inflammatory cytokines and facilitates mononuclear cell adhesion to vascular endothelium $(8,9)$. Elevated levels of $P$. gingivalis LPS appear to present a risk factor for the development of atherosclerosis (10). Furthermore, chronic infusion of $P$. gingivalis LPS increased atherosclerosis formation in $\mathrm{ApoE}^{-/}$mice (11). To this end, the interaction between $P$. gingivalis LPS and macrophages regarding their atherogenic role appears to be reciprocal. On the one hand, many studies have suggested that LPS isolated from $P$. gingivalis activates macrophages to convert into foam cells (12-16). 
On the other hand, only two studies have reported the mechanisms of macrophage-derived foam cell formation stimulated by $P$. gingivalis LPS. Lei et al (12) reported that $P$. gingivalis LPS-induced foam cell formation may be mediated through the enhanced activation of the nuclear factor- $\kappa \mathrm{B}$ pathway. Moreover, Kuramitsu et al (17) suggested that the modification of LDL induced by $P$. gingivalis LPS is likely to promote foam cell formation. In view of the complex nature of cellular lipid level regulation, the detail mechanisms underlying the effect of $P$. gingivalis LPS on foam cell formation remain to be further investigated.

It is well-established that SRs and RCTs dynamically mediate intracellular cholesterol levels (5). Thus, their expression levels are important for foam cell formation. The expression of these mediators may be altered by transcriptional regulation including factors such as activator protein-1 (AP-1) for CD36 $(18,19)$ and HO-1 for ABCA1 $(20)$. Meanwhile, the expression of RCTs may be altered through post-translational regulation which is related to protein stability $(21,22)$. To date, the molecular mechanisms by which $P$. gingivalis LPS influences the expression of RCTs and SRs resulting in increased lipid accumulation in foam cells are still unclear.

The purpose of this study was firstly to examine the effect of $P$. gingivalis LPS on cellular lipid levels in THP-1-derived macrophages; secondly, to investigate the effect of $P$. gingivalis LPS on the expression of SRs and RCTs; and thirdly, to delineate the possible molecular mechanisms involved in $P$. gingivalis LPS-induced changes in the expression of RCTs and SRs as well as foam cell formation.

\section{Materials and methods}

Cells and reagents. The THP-1 human monocyte-derived cell line was obtained from the American Type Culture Collection (ATCC, Manassas, VA, USA), and maintained in RPMI-1640 medium supplemented with $10 \%$ fetal bovine serum (FBS), $20 \mathrm{IU} / \mathrm{ml}$ penicillin and $20 \mathrm{~g} / \mathrm{ml}$ streptomycin. Cells were maintained at $37^{\circ} \mathrm{C}$ in an atmosphere of $5 \% \mathrm{CO}_{2}-95 \%$ room air and differentiated into macrophages by the addition of phorbol 12-myristate 13-acetate (PMA; $200 \mathrm{ng} / \mathrm{ml}$ ) for $48 \mathrm{~h}$. Rabbit anti-c-Fos, rabbit anti-HO-1, rabbit anti-c-Jun antibodies were from Cell Signaling Technology, Inc. (Beverly, MA, USA). Mouse anti-ABCA1, rabbit anti-CD36, rabbit anti-SR-BI, rabbit anti-calpain and rabbit anti-ABCG1 antibodies were obtained from Abcam (Cambridge, MA, USA). Rabbit anti-calpastatin and goat anti-SR-A antibody were purchased from Santa Cruz Biotechnology, Inc. (Santa Cruz, CA, USA). Scrambled and HO-1 small hairpin (sh) RNA were from Shanghai GenePharma Co., Ltd. (Shanghai, China). 3-Dodecanoyl-NBD-cholesterol was obtained from Cayman Chemical Co. (Ann Arbor, MI, USA). PMA was from Sigma (St. Louis, MO, USA). Cholesterol assay kit was obtained from Nanjing Jiancheng Bioengineering Institute (Nanjing, China). Assay kit for calpain activity was supplied by Biovision (Lyon, France).

shRNA transfection. shRNA transfection experiments were conducted as previously described (19). In brief, cell transfections were performed with the SuperFect fragment (Qiagen, Valencia, CA, USA) according to the manufacturer's instruc- tions using scrambled or HO-1 shRNA in a 6-well plate or $50 \mathrm{ml}$ flask. Cells were incubated for $24 \mathrm{~h}$ after transfection and used for the indicated experiments.

Oil Red $O$ staining. Oil Red O staining was used to observe lipid accumulation in foam cells derived from macrophages. After the culture of THP-1-derived macrophages with $50 \mu \mathrm{g} / \mathrm{ml}$ oxLDL in the presence or absence of $P$. ging $i$ valis LPS for $24 \mathrm{~h}$, cells were fixed in $4 \%$ paraformaldehyde and stained with Oil Red $\mathrm{O}$ and hematoxylin. After Oil Red O staining, the density of the lipid content was examined by alcohol extraction. The absorbance at $540 \mathrm{~nm}$ was evaluated by a microplate reader (BioTek Instruments, Inc., Winooski, VT, USA).

Cholesterol efflux assay. Cholesterol efflux experiments were performed as previously described (19). After a 24-h treatment with various concentrations of $P$. gingivalis LPS, the THP-1derived macrophages were incubated with the equilibration of NBD-cholesterol $(1 \mu \mathrm{g} / \mathrm{ml})$ for an additional $6 \mathrm{~h}$ in the presence of $P$. gingivalis LPS. The NBD-cholesterol-labeled cells were incubated in RPMI-1640 medium for $6 \mathrm{~h}$ after washing with PBS. A multilabel counter (Perkin-Elmer Life Sciences, Waltham, MA, USA) was used to detect the fluorescence-labeled cholesterol released from the cells into the medium. Cholesterol efflux was calculated as a percentage of fluorescence in the medium relative to the total amount of fluorescence.

Quantitative real-time polymerase chain reaction ( $q R T-P C R$ ). Total RNA was isolated using RNAiso Plus and was converted into complementary DNA by the PrimeScript RT reagent kit (Perfect Real Time; Takara Bio, Inc., Shiga, Japan). The reaction of qRT-PCR was performed using the $\mathrm{iQ}^{\mathrm{TM}}$ SYBR-Green Supermix (Bio-Rad, Hercules, CA, USA) under the following conditions: $3 \mathrm{~min}$ at $95^{\circ} \mathrm{C}$ for $1 \mathrm{cycle}, 10 \mathrm{sec}$ at $95^{\circ} \mathrm{C}, 30 \mathrm{sec}$ at $60^{\circ} \mathrm{C}$ for 39 cycles, and $95^{\circ} \mathrm{C}$ for $5 \mathrm{sec}$. The following primers were used: GAPDH forward, 5'-GGTGAAGGTCGGTGTGA ACG-3' and reverse, 5'-CTCGCTCCTGGAAGATGGTG-3'; SR-BI forward, 5'-ACCCTAACCCAAAGGAGCAT-3' and reverse, 5'-CACAGCAACGGCAGAACTAC-3'; ABCG1 forward, 5'-GCCTACTACCTGGCAAAGACC-3' and reverse, 5'-GAACAGCACAAAACGCACAG-3'; ABCA1 forward, 5'-CAATGTCAAGGTGTGGTTCAAT-3' and reverse, 5'-GC TGCTGTTTAGTGAGGTTCAA-3'; SR-A forward, 5'-TCCT TGATTTCGTCAGTCCAG-3' and reverse, 5'-CCTCCTGTT GCTTTGCTGTAG-3'; CD36 forward, 5'-TCGCTTCCACAT TTCCTACAT-3' and reverse, 5'-CCCAGTCTCATTTAGCC ACAG-3'.

Western blot analysis. The methods for nuclear extracts and western blot analysis were described in our previous study (19). THP-1-derived macrophages were harvested and lysed with $180 \mu$ l RIPA lysis buffer (Beyotime, Jiangsu, China). Proteins from total cell lysates, measured using the bicinchoninic acid protein assay kit (Biomed Biotech Co., Ltd., Beijing, China), were boiled in 5X SDS-sample buffer for $10 \mathrm{~min}$, separated on $8-12 \%$ SDS-polyacrylamide minigels and then transferred onto nitrocellulose membranes (Amersham, Buckinghamshire, UK). After blocking with 5\% non-fat milk in PBST, the 
$\mathrm{A}_{\mathrm{a}}$

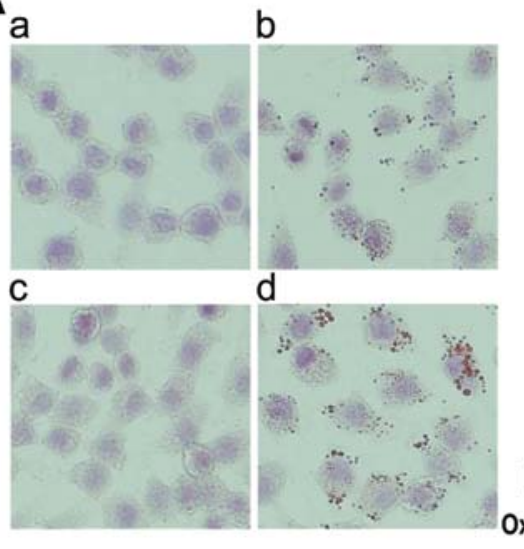

B

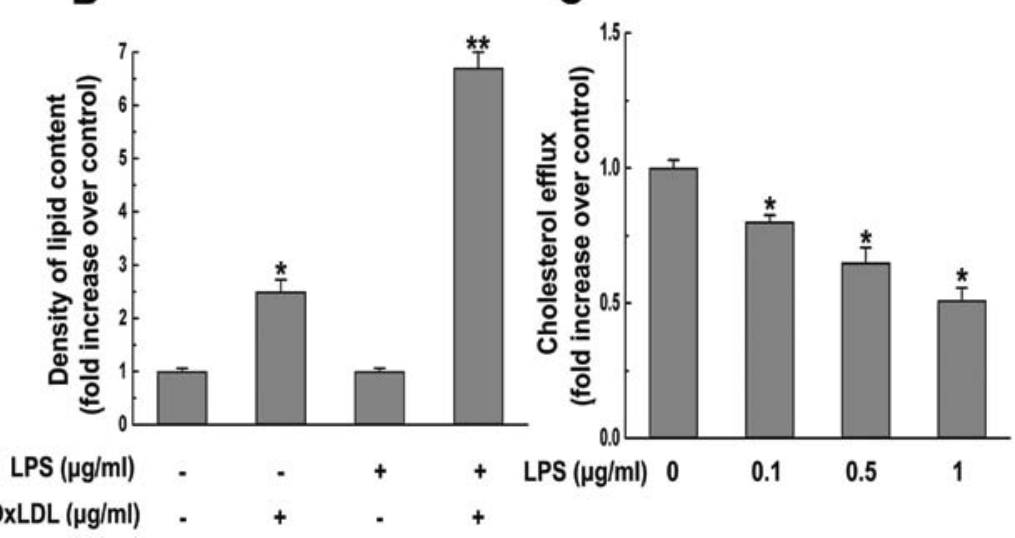

Figure 1. P. gingivalis LPS promotes oxLDL-mediated lipid accumulation via inhibition of cholesterol efflux in macrophages. THP-1-derived macrophages were co-treated with $P$. gingivalis LPS $(0.1 \mu \mathrm{g} / \mathrm{ml})$ and oxLDL $(50 \mathrm{mg} / \mathrm{ml})$ for $24 \mathrm{~h}$. (A) The cells subjected to various treatments were fixed and then stained with Oil Red O. (a) Control group; (b) oxLDL-treated group; (c) P. gingivalis LPS-treated group; (d) oxLDL plus $P$. gingivalis LPS group. Magnification, x400. (B) The density of the lipid content was measured by extracting the Oil Red O stained lipid and measuring the absorbance at 540 nm. (C) THP-1-derived macrophages were pretreated with various concentrations of $P$. gingivalis LPS $(0,0.1,0.5,1 \mu \mathrm{g} / \mathrm{ml})$ for $24 \mathrm{~h}$ and then treated with NBD-cholesterol $(1 \mu \mathrm{g} /$ $\mathrm{ml}$ ) for an additional $6 \mathrm{~h}$ in the presence of $P$. gingivalis LPS. Cholesterol efflux was expressed as the percentage of fluorescence in the medium relative to total fluorescence. The data are representative of 3 independent experiments (means $\pm \mathrm{SEM})$. ${ }^{*} \mathrm{P}<0.05$ compared with control, ${ }^{* * *} \mathrm{P}<0.05$ compared with the oxLDL-treated group.

membrane was incubated with primary antibodies overnight at $4^{\circ} \mathrm{C}$. The protein expression was detected by an enhanced chemiluminescence kit (ECL; Perkin-Elmer Life Sciences) and densitometric analysis was performed using the 720 BK/01837 System (Bio-Rad).

Immunoprecipitation. Cell lysates containing equal amounts of protein $(1,000 \mathrm{mg})$ from THP-1-derived macrophages treated with or without $P$. gingivalis LPS for $24 \mathrm{~h}$ were incubated with the specific primary antibody overnight at $4^{\circ} \mathrm{C}$, and then with protein A/G-Sepharose for $2 \mathrm{~h}$. Immune complexes were collected and eluted in lysis buffer. Eluted protein samples were then boiled in SDS-PAGE loading buffer for subsequent western blot analysis.

Measurement of calpain activity. Calpain activity was measured as previously described (20). Briefly, cellular lysates (100 mg) were mixed with reaction buffer and fluorogenic substrate Ac-LLY-AFC. The level of released AFC was measured over $1 \mathrm{~h}$ at $37^{\circ} \mathrm{C}$ by fluorometry using 400-nm excitation and 505-nm emission filters.

Statistical analysis. Data are presented as the means \pm SEM and were analyzed using one-way analysis of variance (ANOVA), and the Newman-Keuls test was used to determine any significant differences identified by ANOVA. Differences were considered statistically significant at $\mathrm{P}<0.05$. All experiments were performed at least 3 times.

\section{Results}

P. gingivalis LPS enhances intracellular lipid accumulation and decreases cholesterol efflux in oxLDL-induced macrophages. We investigated the effect of $P$. gingivalis LPS on oxLDL-induced foam cell formation. Treatment with oxLDL caused an increase in lipid accumulation and a decrease in cellular cholesterol efflux. Notably, the effects of oxLDL on lipid accumulation and cholesterol efflux were significantly exacerbated by additional treatment with $P$. gingivalis LPS (Fig. 1). These results indicate that $P$. gingivalis LPS possesses pro-atherogenic activities during the formation of foam cells.

P. gingivalis LPS decreases the expression of ABCA1, but increases the expression of CD36 in macrophages. To investigate the mechanisms underlying the exacerbation of foam cell formation by $P$. gingivalis LPS, the effects of $P$. gingivalis LPS on RCTs and SRs were examined. Treatment with $P$. gingivalis LPS at various concentrations $(0.1,0.5$ and $1 \mu \mathrm{g} / \mathrm{ml}$ ) for $24 \mathrm{~h}$ dose-dependently decreased the mRNA and protein expression of ABCA1 without affecting the expression of ABCG1 and SR-BI (Figs. 2 and 3). Moreover, the expression of CD36 was significantly increased at the protein and mRNA levels in response to $P$. gingivalis LPS treatment, while the protein and mRNA levels of SR-A were not altered by $P$. gingivalis LPS incubation (Figs. 2 and 3).

$P$. gingivalis LPS-induced CD36 expression is mediated by the $c$-Jun-AP-1 pathway. It has been reported that AP-1 (c-Jun and c-Fos, 2 key subunits of AP-1) activity contributes to the fate of the cell after $P$. gingivalis LPS treatment (23). Thus, we detected the possibility that $P$. gingivalis LPS-induced CD36 expression in macrophages is mediated by the AP-1 pathway. Our results revealed that $P$. gingivalis LPS treatment had no effect on the nuclear expression of c-Fos, while $P$. gingivalis LPS treatment of macrophages caused dose-dependent increases in nuclear c-Jun expression (Fig. 4A). Additionally, c-Jun N-terminal kinase (JNK) inhibitor, SP600125, which is a potent, cell-permeable selective and reversible inhibitor of JNK1, JNK2 and JNK3, blocked $P$. gingivalis LPS-induced CD36 expression (Fig. 4B). Collectively, these results suggest that induction of CD36 expression and subsequent exacerbation of foam cell formation by $P$. gingivalis LPS are partly c-Jun-AP-1-dependent. 

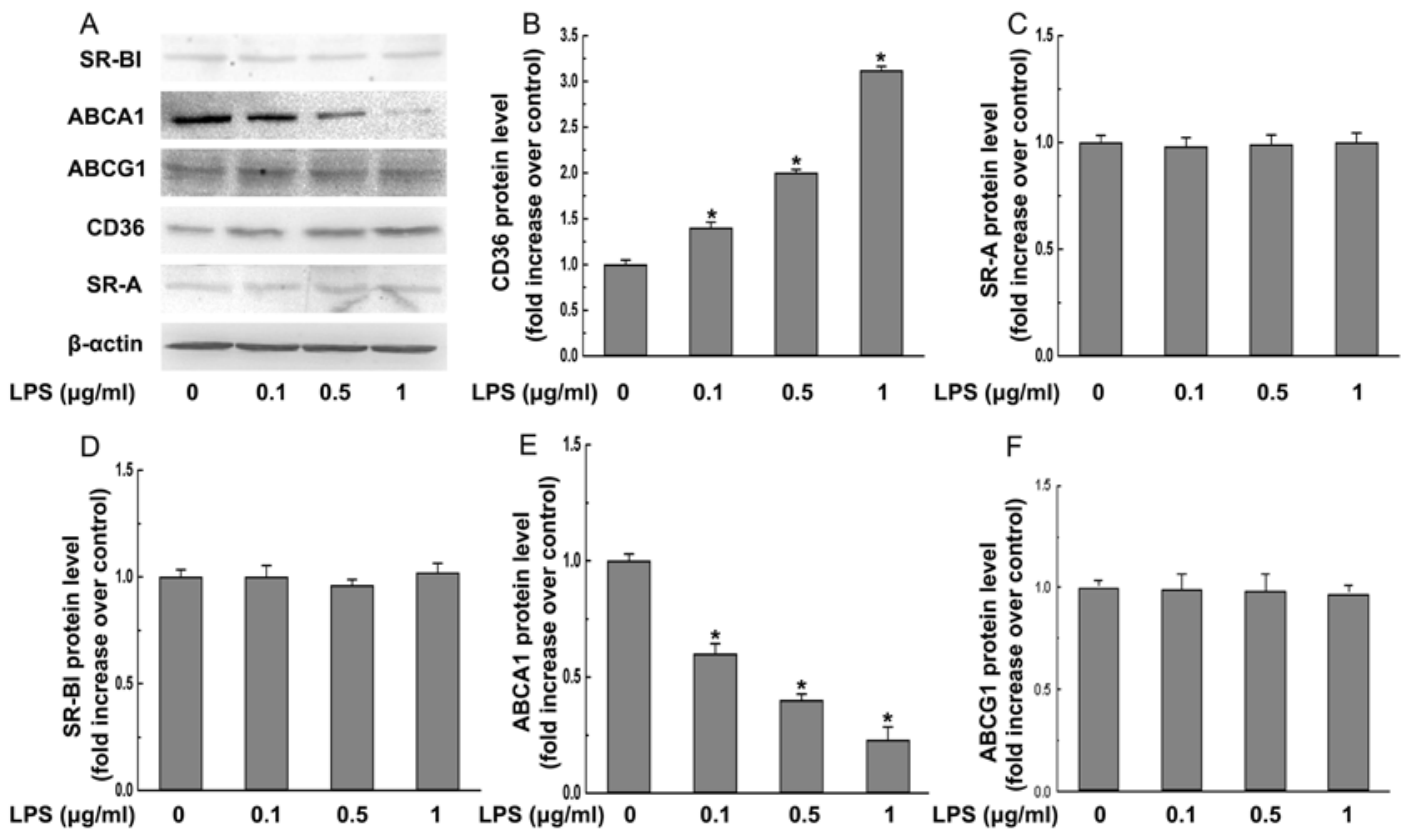

Figure 2. P. gingivalis LPS (LPS) decreases protein expression of ABCA1, but increases protein expression of CD36. (A) THP-1-derived macrophages were treated with the indicated concentrations $(0,0.1,0.5,1 \mu \mathrm{g} / \mathrm{ml})$ of $P$. gingivalis LPS for $24 \mathrm{~h}$ and the protein levels of SR-A, CD36, SR-BI, ABCA1, ABCG1, or $\beta$-actin were determined by western blotting. The relative protein levels of (B) CD36, (C) SR-A, (D) SR-BI, (E) ABCA1, (F) ABCG1 are presented as means \pm SEM of the optical density from 3 separated experiments, ${ }^{*} \mathrm{P}<0.05$ compared with the control.
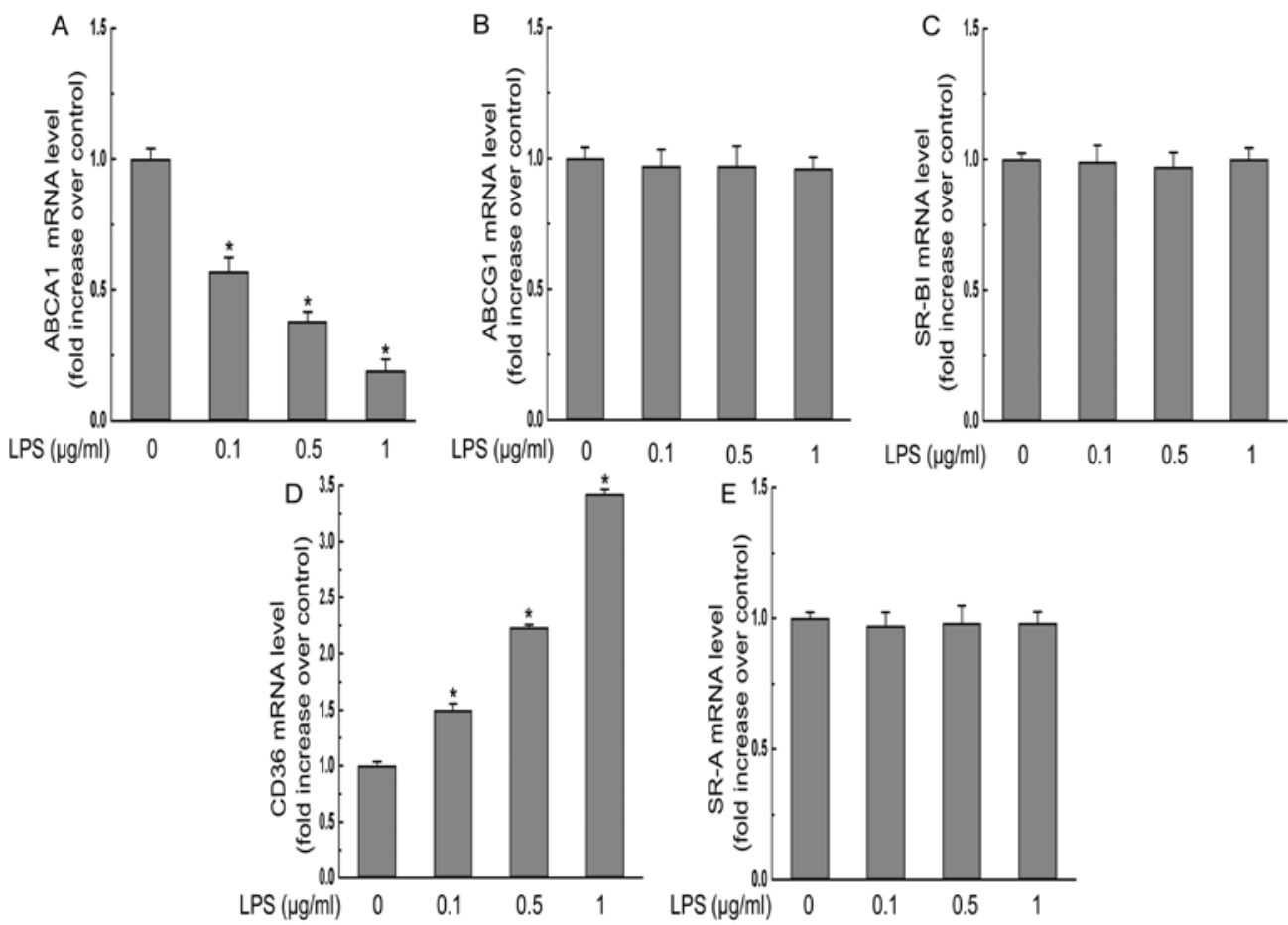
Figure 3. $P$. gingivalis LPS (LPS) decreases the mRNA expression of ABCA1, but increases the mRNA expression of CD36 in macrophages. THP-1-derived
macrophages were treated with $P$. gingivalis LPS $(0,0.1,0.5,1 \mu \mathrm{g} / \mathrm{ml})$ for $24 \mathrm{~h}$. After treatment, total RNA was extracted and then subjected to $\mathrm{qRT}$-PCR to detect the mRNA expression of (A) ABCA1, (B) ABCG1, (C) SR-BI, (D) CD36, (E) SR-A. The data are representative of 3 independent experiments (means $\pm \mathrm{SEM}) .{ }^{*} \mathrm{P}<0.05$ compared with the control.

P. gingivalis LPS decreases the stability of ABCA1 protein by increasing calpain activity. Further analysis of the protein stability of ABCA1 showed that, in the presence of CHX (an inhibitor of de novo protein synthesis), the degradation profile of $\mathrm{ABCA} 1$ protein during a 12 -h treatment with $P$. gingivalis LPS was more rapid than in the group without
P. gingivalis LPS (Fig. 5A and B). We further investigated the involvement of calpain, a protease involved in ABCA1 proteolysis (20). As shown in Fig. 5C, $P$. gingivalis LPS incubation enhanced the calpain activity in a dose-dependent manner. However, the expression of calpain and calpastatin (the endogenous inhibitor for calpain) were not altered by 

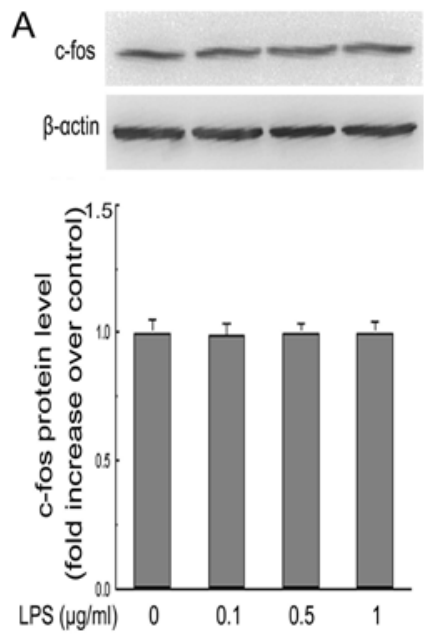
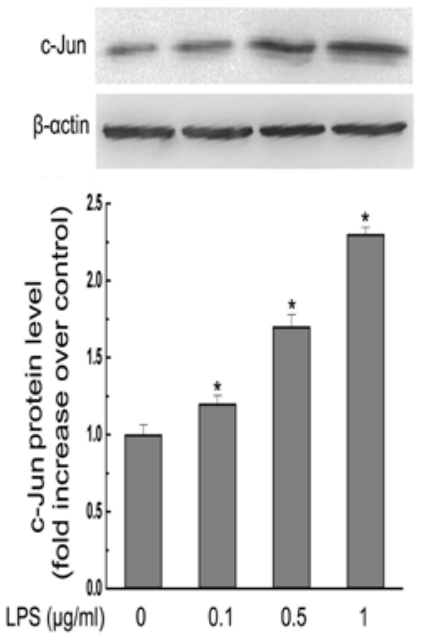

\section{B}
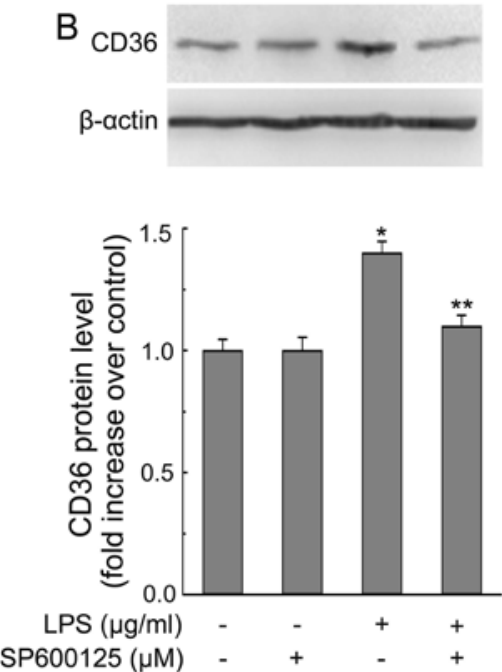

Figure 4. Nuclear translocation of c-Jun is involved in P. gingivalis LPS (LPS)-mediated increase of CD36 expression in macrophages. (A) THP-1-derived macrophages were treated with $P$. gingivalis LPS $(0,0.1,0.5,1 \mu \mathrm{g} / \mathrm{ml})$ for $6 \mathrm{~h}$, nuclear extracts were prepared and the protein level of c-Fos and c-Jun was detected by western blotting. (B) Macrophages were pre-treated with SP600125 $(10 \mu \mathrm{M})$ for $1 \mathrm{~h}$, and then treated in the presence or absence of $P$. gingivalis LPS $(0.1 \mu \mathrm{g} / \mathrm{ml})$ for $24 \mathrm{~h}$. Protein expression of CD36 was determined by western blotting. The data are representative of 3 independent experiments (means $\pm \mathrm{SEM}) .{ }^{*} \mathrm{P}<0.05$ compared with the control; ${ }^{* *} \mathrm{P}<0.05$ compared with the $P$. gingivalis LPS-treated group.

A

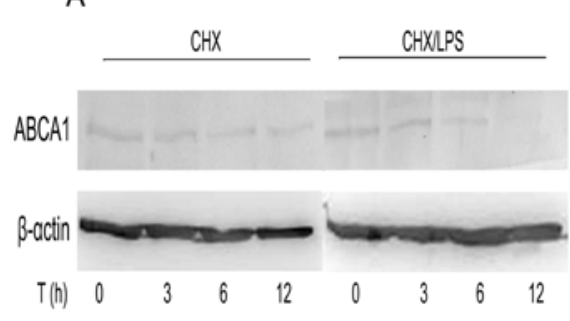

\section{D}
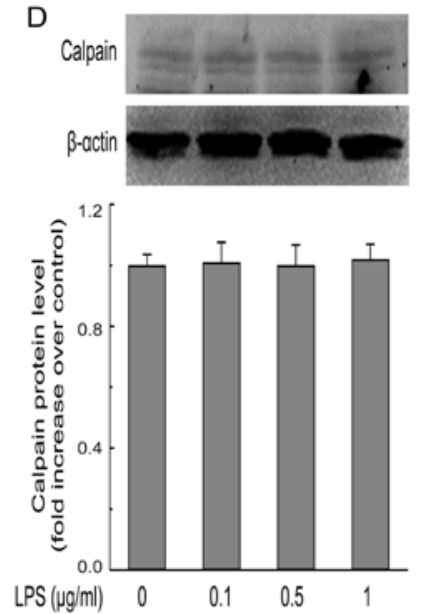

B

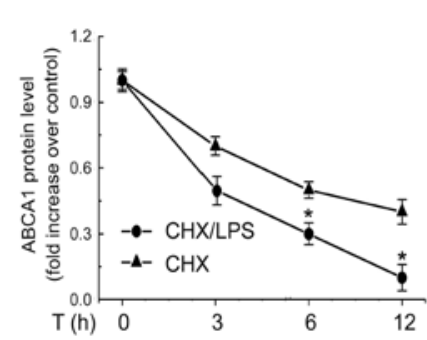

2
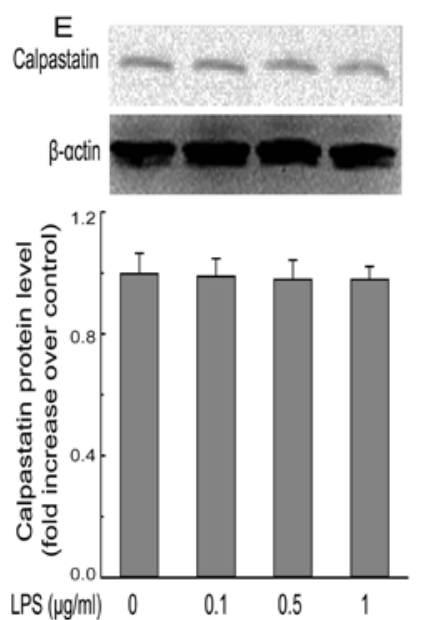

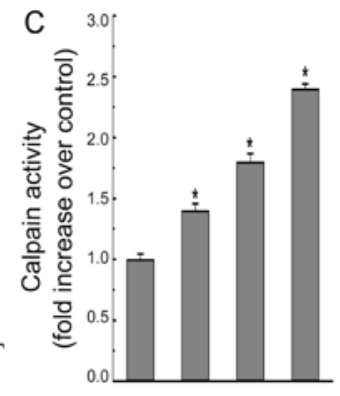

$\mathrm{F}$

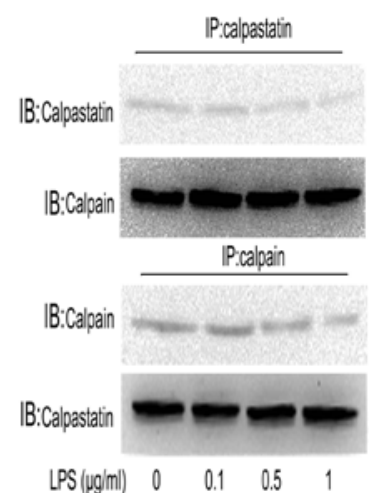

$\operatorname{LPS}(\mu g / m)) \quad 0 \quad 0.1 \quad 0.5 \quad 1$

Figure 5. P. gingivalis LPS decreases the stability of ABCA1 protein and enhances calpain activity. (A and B) THP-1-derived macrophages were incubated with or without $P$. gingivalis LPS $(0.1 \mu \mathrm{g} / \mathrm{ml})$ in the presence of cyclohexamide (CHX; $2 \mathrm{mg} / \mathrm{ml})$ for the indicated times. Cellular lysates were subjected to western blotting to determine the level of ABCA1 or $\beta$-actin. (C) Macrophages were treated with the indicated concentrations of $P$. gingivalis LPS for $24 \mathrm{~h}$ and calpain activity was determined. (D and E) Cellular lysates were subjected to western blotting to determine the expression of calpain, calpastatin, or $\beta$-actin. (F) Cellular lysates were immunoprecipitated (IP) with anti-calpain or anti-calpastatin antibody and then immuno-probed (IB) with anti-calpastatin or anti-calpain antibody. The data are representative of 3 independent experiments (means $\pm \mathrm{SEM}$ ). ${ }^{*} \mathrm{P}<0.05$ compared with the control.

$P$. gingivalis LPS (Fig. 5D and E). Obviously, the enhanced calpain activity resulted from a decrease in the protein interaction between calpain and calpastatin (Fig. 5F).
The enhanced effect of $P$. gingivalis LPS on foam cell formation is mediated by HO-1. We determined the role of HO-1 in $P$. gingivalis LPS-mediated exacerbation in foam cells. The 

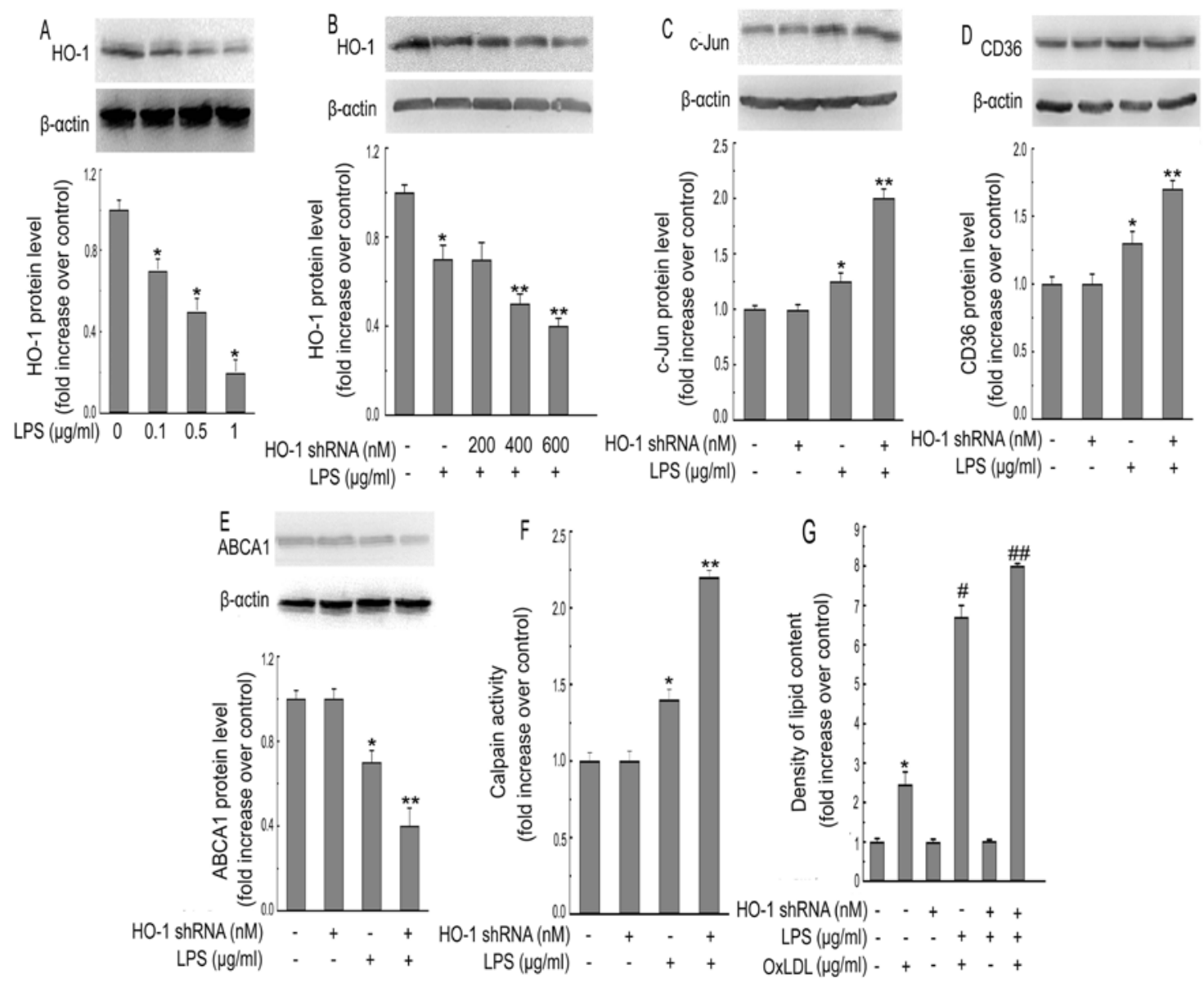

Figure 6. P. gingivalis LPS (LPS)-induced atherogenic effects in macrophages are mediated by HO-1. (A) THP-1-derived macrophages were incubated with various concentrations $(0,0.1,0.5,1 \mu \mathrm{g} / \mathrm{ml})$ of $P$. gingivalis LPS for $12 \mathrm{~h}$. Western blotting was used to detect the protein expression of HO-1 following treatment. (B) THP-1-derived macrophages were transfected with various concentrations of HO-1 shRNA (200, $400,600 \mathrm{nM})$ for $24 \mathrm{~h}$, followed by $P$. gingivalis LPS treatment $(0.1 \mu \mathrm{g} / \mathrm{ml})$ for an additional $12 \mathrm{~h}$. Protein expression of HO-1 and $\beta$-actin was measured by western blotting. (C-E) Macrophages were pre-treated with HO-1 shRNA $(600 \mathrm{nM})$ for $24 \mathrm{~h}$, followed by P. gingivalis LPS for an additional $6 \mathrm{~h}(\mathrm{C})$ or $24 \mathrm{~h}$ (D and E). Protein levels of c-Jun, $\beta$-actin, CD36 and ABCA1 were determined by western blotting. (F) Calpain activity was measured by using an enzymatic method. (G) OxLDL-induced lipid accumulation was measured by alcohol extraction. The data are representative of 3 independent experiments (means $\pm \mathrm{SEM}$ ). ${ }^{*}<0.05$ compared with the control, ${ }^{* *} \mathrm{P}<0.05$ compared with the $P$. gingivalis LPS-treated group. ${ }^{\#} \mathrm{P}<0.05$ compared with the OxLDL-treated group. ${ }^{\# \#} \mathrm{P}<0.05$ compared with the $P$. gingivalis LPS plus OxLDL group.

protein level of HO-1 in macrophages was dose-dependently decreased in response to $P$. gingivalis LPS as measured by western blot analysis (Fig. 6A). Moreover, transfection of the HO-1 shRNA for gene knockdown resulted in promotion of the $P$. gingivalis LPS-induced effect on HO-1 expression (Fig. 6B), whereas transfection with the corresponding scrambled shRNA failed to do so. Additionally, transfection with HO-1 shRNA augmented the $P$. gingivalis LPS-mediated effects on the upregulation of c-Jun (Fig. 6C), and CD36 protein expression (Fig. 6D), downregulation of ABCA1 protein expression (Fig. 6E), promotion of calpain activity (Fig. 6F), and augmentation of lipid accumulation (Fig. 6G) in THP-1derived macrophages, indicating the crucial role of HO-1 in the exacerbating effects by $P$. gingivalis LPS.

\section{Discussion}

Emerging data reveal that $P$. gingivalis LPS may have an atherogenic effect on promoting intracellular lipid accumulation during the formation of macrophage-derived foam cells $(12,17)$. In the present study, our results indicated that $P$. gingivalis LPS indeed augmented the uptake of oxLDL in THP-1-derived macrophages, consistent with previous studies $(12,17)$. We then used this experimental model to explore the molecular mechanisms underlying $P$. gingivalis LPS-induced promotion of foam cell formation. Our data showed that $P$. gingivalis LPS markedly increased the protein and mRNA expression of CD36, without changing the expression of SR-A. The importance of CD36 and SR-A in atherogenesis and foam cell formation is well established (24). The genetic inactivation of CD36 has previously been shown to reduce oxLDL uptake in vitro and in atherosclerotic lesions in mice (25), strongly supporting a pro-atherogenic role for CD36. The expression of CD36 in macrophages is also known to be upregulated by inflammatory cytokines or stimuli that have an atherogenic role (26). In view of CD36 function, it may be concluded that the increase in CD36 expression followed by $P$. gingivalis LPS treatment may contribute to the exacerbated lipid accumulation and subsequent promotion of foam cell formation.

In the present study, we demonstrated for the first time that c-Jun-AP-1 is the key transcriptional factor for $P$. gingivalis LPS-induced upregulation of CD36. This notion is also 
supported by the finding that inhibition of AP-1 suppressed the upregulation of CD36 by $P$. gingivalis LPS. A recent study (23) reported that $P$. gingivalis LPS, at a similar concentration, activated both c-Jun and c-Fos, two subunit of AP-1 in RAW 264.7 cells, a mouse cell line. The discrepancy between this and that study (23) could be due to the differences in cell types.

In addition to its effects on CD36 expression, our results further demonstrated that $P$. gingivalis LPS decreased the mRNA and protein levels of ABCA1 without affecting the protein and mRNA expression of ABCG1 or SR-BI. Cholesterol efflux in macrophages is mainly regulated by ABCA1 (27). The results of the present study confirmed a recent in vivo study that the ABCA1 mRNA level in the aorta was significantly reduced in long-term $P$. gingivalis-infected mice (28). The importance of ABCA1 in maintaining cholesterol homeostasis in macrophages is well established (29). Additionally, the expression of ABCA1 is well known to be downregulated by various inflammatory cytokines (30). In view of the function of ABCA1 function, the decreased expression of ABCA1 by $P$. gingivalis LPS detected in the present study is also likely to contribute to foam cell formation.

Furthermore, we provide evidence for the involvement of calpain protease in $P$. gingivalis LPS-decreased post-transcriptional regulation of $\mathrm{ABCA} 1$, as revealed by increased calpain activity and decreased interaction of calpain and calpastatin, in response to $P$. gingivalis LPS treatment. In fact, the critical role of calpain in the stabilization of ABCA1 protein is well established (31,32). Moreover, protein kinase C (PKC) phosphorylates and stabilizes ABCA1 through inhibition of its degradation mediated by calpain (33). The involvement of this pathway in the $P$. gingivalis LPS-induced downregulation of ABCA1 protein degradation warrants further investigation.

More importantly, our results suggest that the decreased expression of ABCA1, upregulated expression of CD36 and increased c-Jun/AP-1 nuclear translocation induced by $P$. gingivalis LPS is accompanied by decreased HO-1 expression. This finding was further supported by the results from the western blot analysis, in which the $P$. gingivalis LPS-mediated upregulation of CD36, downregulation of ABCA1, and promotion of c-Jun/AP-1 nuclear translocation was augmented by the knockdown of the HO-1 gene using shRNA. In addition, the inhibition of HO-1 exacerbated the $P$. gingivalis LPS effects on the upregulation of lipid accumulation and calpain activity in the macrophages. These results suggest that the decreased expression of HO-1 is required for the decreased expression of ABCA1, upregulated expression of CD36 and increased c-Jun/AP-1 nuclear translocation induced by $P$. gingivalis LPS. Our findings are in agreement with reports that deletion of HO-1 leads to the exacerbation of atherosclerosis and foam cell formation (34). A number of studies have reported that bilirubin or carbon monoxide regulates the antioxidative or anti-inflammatory action of HO-1 $(35,36)$. The involvement of this pathway in the $P$. gingivalis LPS-induced promotion of foam cell formation warrants further investigation.

In summary, our study provides new insight into the crucial role of HO-1 in the $P$. gingivalis LPS-mediated atherogenic effects in macrophages, which exacerbates lipid accumulation in oxLDL-induced foam cells by a decrease in cholesterol efflux. The cholesterol efflux regulated by $P$. gingivalis LPS is via transcriptional upregulation of CD36 expression and transcriptional and post-transcriptional downregulation of ABCA1 expression. The results of the present study provide a potential mechanism for the contribution of $P$. gingivalis LPS to atherogenesis and shed light on the underlying mechanism by which periodontitis affects atherosclerosis and subsequent coronary heart disease in humans.

\section{Acknowledgements}

This investigation was supported by a grant from the Public Health Bureau of Chongqing China (grant no. 2012-2-137).

\section{References}

1. Xue JH, Yuan Z, Wu Y, et al: High glucose promotes intracellular lipid accumulation in vascular smooth muscle cells by impairing cholesterol influx and efflux balance. Cardiovasc Res 86: 141-150, 2010.

2. Li AC and Glass CK: The macrophage foam cell as a target for therapeutic intervention. Nat Med 8: 1235-1242, 2002.

3. Kleemann R, Zadelaar S and Kooistra T: Cytokines and atherosclerosis: a comprehensive review of studies in mice. Cardiovasc Res 79: 360-376, 2008.

4. Lin CY, Lee TS, Chen CC, Chang CA, Lin YJ, Hsu YP and Ho LT: Endothelin-1 exacerbates lipid accumulation by increasing the protein degradation of the ATP-binding cassette transporter G1 in macrophages. J Cell Physiol 226: 2198-2205, 2011.

5. Cheng LC, Su KH, Kou YR, Shyue SK, et al: $\alpha$-Lipoic acid ameliorates foam cell formation via liver $\mathrm{X}$ receptor $\alpha$-dependent upregulation of ATP-binding cassette transporters A1 and G1. Free Radic Biol Med 50: 47-54, 2011.

6. Park YM, R Kashyap S, Major J and Silverstein RL: Insulin promotes macrophage foam cell formation: potential implications in diabetes-related atherosclerosis. Lab Invest 92: 1171-1180, 2012.

7. Morimoto Y, Kikuchi K, Ito T, et al: MK615 attenuates Porphyromonas gingivalis lipopolysaccharide-induced proinflammatory cytokine release via MAPK inactivation in murine macrophage-like RAW264.7 cells. Biochem Biophys Res Commun 389: 90-94, 2009.

8. Triantafilou M, Gamper FG, Lepper PM, et al: Lipopolysaccharides from atherosclerosis-associated bacteria antagonize TLR4, induce formation of TLR2/1/CD36 complexes in lipid rafts and trigger TLR2-induced inflammatory responses in human vascular endothelial cells. Cell Microbiol 9: 2030-2039, 2007.

9. Nakamura N, Yoshida M, Umeda M, et al: Extended exposure of lipopolysaccharide fraction from Porphyromonas gingivalis facilitates mononuclear cell adhesion to vascular endothelium via Toll-like receptor-2 dependent mechanism. Atherosclerosis 196: 59-67, 2008.

10. Kiechl S, Egger G, Mayr M, et al: Chronic infections and the risk of carotid atherosclerosis: prospective results from a large population study. Circulation 103: 1064-1070, 2001.

11. Gitlin JM and Loftin CD: Cyclooxygenase-2 inhibition increases lipopolysaccharide-induced atherosclerosis in mice. Cardiovasc Res 81: 400-407, 2009.

12. Lei L, Li H, Yan F, Li Y and Xiao Y: Porphyromonas gingivalis lipopolysaccharide alters atherosclerotic-related gene expression in oxidized low-density-lipoprotein-induced macrophages and foam cells. J Periodontal Res 46: 427-437, 2011.

13. Pussinen PJ and Mattila K: Periodontal infections and atherosclerosis: mere associations? Curr Opin Lipidol 15: 583-588, 2004.

14. Qi M, Miyakawa H and Kuramitsu HK: Porphyromonas gingivalis induces murine macrophage foam cell formation. Microb Pathog 35: 259-267, 2003.

15. Kuramitsu HK, Kang IC and Qi M: Interactions of Porphyromonas gingivalis with host cells: implications for cardiovascular diseases. J Periodontol 74: 85-89, 2003.

16. Kuramitsu HK, Qi M, Kang IC and Chen W: Role for periodontal bacteria in cardiovascular diseases. Ann Periodontol 6: 41-47, 2001.

17. Kuramitsu HK, Miyakawa H, Qi M and Kang IC: Cellular responses to oral pathogens. Ann Periodontol 7: 90-94, 2002. 
18. Katayama I, Hotokezaka Y, Matsuyama T, Sumi T and Nakamura T: Ionizing radiation induces macrophage foam cell formation and aggregation through JNK-dependent activation of CD36 scavenger receptors. Int J Radiat Oncol Biol Phys 70: 835-846, 2008

19. Li XY, Kong LX, Li J, He HX and Zhou YD: Kaempferol suppresses lipid accumulation in macrophages through the downregulation of cluster of differentiation 36 and the upregulation of scavenger receptor class B type I and ATP-binding cassette transporters A1 and G1. Int J Mol Med 31: 331-338, 2013.

20. Tsai JY, Su KH, Shyue SK, et al: EGb761 ameliorates the formation of foam cells by regulating the expression of SR-A and ABCA1: role of haem oxygenase-1. Cardiovasc Res 88: 415-423, 2010.

21. Feng B and Tabas I: ABCA1-mediated cholesterol efflux is defective in free cholesterol-loaded macrophages. Mechanism involves enhanced ABCA1 degradation in a process requiring full NPC1 activity. J Biol Chem 277: 43271-43280, 2002.

22. Wang Y and Oram JF: Unsaturated fatty acids inhibit cholesterol efflux from macrophages by increasing degradation of ATP-binding cassette transporter A1. J Biol Chem 277: 5692-5697, 2002.

23. Kim HS, Park SY, Kim EK, Ryu EY, Kim YH, Park G and Lee SJ: Acanthopanax senticosus has a heme oxygenase-1 signaling-dependent effect on Porphyromonas gingivalis lipopolysaccharide-stimulated macrophages. J Ethnopharmacol 142: 819-828, 2012.

24. de Winther MP and Hofker MH: Scavenging new insights into atherogenesis. J Clin Invest 105: 1039-1041, 2000.

25. Kunjathoor VV, Febbraio M, Podrez EA, et al: Scavenger receptors class A-I/II and CD36 are the principal receptors responsible for the uptake of modified low density lipoprotein leading to lipid loading in macrophages. J Biol Chem 277: 49982-49988, 2002

26. Ortiz-Masià D, Díez I, Calatayud S, et al: Induction of CD36 and thrombospondin-1 in macrophages by hypoxia-inducible factor 1 and its relevance in the inflammatory process. PLoS One 7: e48535, 2012.
27. Brooks-Wilson A, Marcil M, Clee SM, et al: Mutations in ABC1 in Tangier disease and familial high-density lipoprotein deficiency. Nat Genet 22: 336-345, 1999.

28. Maekawa T, Takahashi N, Tabeta K, et al: Chronic oral infection with Porphyromonas gingivalis accelerates atheroma formation by shifting the lipid profile. PLoS One 6: e20240, 2011.

29. Oram JF and Heinecke JW: ATP-binding cassette transporter A1: a cell cholesterol exporter that protects against cardiovascular disease. Physiol Rev 85: 1343-1372, 2005.

30. Yin K, Liao DF and Tang CK: ATP-binding membrane cassette transporter A1 (ABCA1): a possible link between inflammation and reverse cholesterol transport. Mol Med 16: 438-449, 2010.

31. Martinez LO, Agerholm-Larsen B, Wang N, Chen W and Tall AR: Phosphorylation of a pest sequence in ABCA1 promotes calpain degradation and is reversed by ApoA-I. J Biol Chem 278: 37368-37374, 2003.

32. Arakawa R and Yokoyama S: Helical apolipoproteins stabilize ATP-binding cassette transporter A1 by protecting it from thiol protease-mediated degradation. J Biol Chem 277: 22426-22429, 2002.

33. Liu XY, Lu Q, Ouyang XP, et al: Apelin-13 increases expression of ATP-binding cassette transporter A1 via activating protein kinase $\mathrm{C} \alpha$ signaling in THP-1 macrophage-derived foam cells. Atherosclerosis 226: 398-407, 2013.

34. Orozco LD, Kapturczak MH, Barajas B, et al: Heme oxygenase-1 expression in macrophages plays a beneficial role in atherosclerosis. Circ Res 100: 1703-1711, 2007.

35. Idriss NK, Blann AD and Lip GY: Hemoxygenase-1 in cardiovascular disease. J Am Coll Cardiol 52: 971-978, 2008.

36. Ishikawa K, Sugawara D, Wang Xp, Suzuki K, Itabe H, Maruyama Y and Lusis AJ: Heme oxygenase-1 inhibits atherosclerotic lesion formation in ldl-receptor knockout mice. Circ Res 88: 506-512, 2001. 